\title{
Der Steppeniltis (Mustela [Putorius] eversmanni soergeli ÉHIK) in der Niederterrasse der Leine und seine klimageschichtliche Bedeutung
}

\author{
Von Otto Sickenberg, Hannover
}

Mit 2 Abbildungen und 2 Tabellen

$\mathrm{Z}$ us a m me $\mathrm{n}$ a ssung. Ein aus Kiesen und Sanden der Leine-Niederterrasse gebaggerter Schädel eines Steppeniltis (Mustela [Putorius] eversmanni soergeli ÉHIK) wird zum Anlaß genommen, die stratigraphische und ökologische Aussagekraft dieser Iltis-Art zu überprüfen. M. eversmanni ist in Mitteleuropa bislang mit Sicherheit nur aus verschiedenen Zeitabschnitten des Würm bekannt geworden, nie aus älteren oder jüngeren Schichten. In der Gegenwart ist der Lebensraum des Steppeniltis ziemlich streng an die Halb- bzw. Kultursteppe und an die Vollsteppe gebunden, wobei die wärmeren Teile des eurasiatischen Steppengürtels bevorzugt werden. Auch während des Würm kann die Art nicht unter wesentlich anderen Umweltsverhältnissen gelebt haben. Es wird daraus gefolgert, daß es auch in den Stadialen nicht nur trocken-kalte, sondern auch trockenwärmere Phasen gegeben hat.

$\mathrm{S}$ u $\mathrm{m} \mathrm{m}$ a $\mathrm{r}$ y. A skull of Mustela (Putorius) eversmanni soergeli ÉHI, discovered by a dredger working in gravels and sands of the Lower-Terrace of the River Leine, is used as a test of the reliability of this species in stratigraphy, and as an ecological indicator. Up to the present time M. eversmanni has been found in Central Europe with certainly only in different periods from the Würm Ice-Age and never in stratas older or younger than the Würm. Its present distribution is rather clothely tied to the semi- and cultivated steppe-lands and to the true steppes, but above all, to the warmer parts of the Eurasian steppe region. In the Würm Ice-Age, the species will not have lived in conditions essentially different from these. From this the conclusion has been drawn that durings the stadials there were not only dry-cold but also dry-warmer phases.

\section{Einleitung}

Die Firma „Heidekies K.G.“ betreibt bei Meitze nördlich von Hannover eine Naßbaggerei, in der Kiese und Sande der Leine-Niederterrasse gefördert werden. Der Betriebsführer, Herr WAGNER, ist bedacht, anfallendes Knochenmaterial aufzusammeln und einer wissenschaftlichen Bearbeitung zuzuführen. Unter dem Baggergut des Jahres 1967 befand sich auch der Schädel eines Steppeniltis (Mustela [Putorius] eversmanni soergeli ÉHIK). Herrn WAGNER ist dafür herzlich zu danken, daß er dieses Stück der Sammlung des Niedersächsischen Landesamtes für Bodenforschung, Hannover-Buchholz, freundlicherweise überließ. Das Exemplar trägt die Sammlungsnummer qd 15. Dieser Fund ist der nordwestlichste seiner Art in Europa. Dies rechtfertigt seine Veröffentlichung. Zugleich bietet sich ein Anlaß, die klimageschichtliche Bedeutung des Auftretens von $M$. eversmanni zu untersuchen.

Diese Studie sei dem Altmeister der deutschen Quartärforschung, Herrn Prof. Dr. P. Woldstedt mit den allerbesten Wünschen gewidmet.

\section{Fundort und Fundlager und Begleitfauna}

Fundort ist die Kiesgrube der „Heidekies K.G. “ bei Meitze (TK 25, Nr. 3424, Mellendorf, R.: 53 550, H.: 26140 ). 
Das durch die Kiesförderung (Naßbaggerei) erschlossene Profil ist nach LANG (1967):

Holozän
Pleistozän $\left\{\begin{array}{rr}1 & \mathrm{~m} \\ 4-7 & \mathrm{~m} \\ 4-6 & \mathrm{~m} \\ 0,1 \mathrm{~m}\end{array}\right.$

$\downarrow$
Flug- und Dünensande

Grobsande, nach oben mittelsandig

sandige Kiese

humoser Schluff (an einigen Stellen, aber nicht überall in der Umgebung erbohrt, ob im Kiesgrubenbereich vorhanden daher fraglich)

Kiese (nicht in Förderung, Beschaffenheit und Mächtigkeit, daher unbekannt)

Das Fluviatil ist eine Ablagerung der Leine und entspricht ihrer Niederterrasse (LANG 1967). Da die Begleitfauna über die genauere Altersstellung innerhalb der Weichselkaltzeit keine Aussagen erlaubt, ergeben sich Anhaltspunkte nur aus der Untersuchung von Torfballen, die gelegentlich zusammen mit den Kiesen gefördert werden. Möglicherweise entstammen jene dem liegenden Schluffband oder anderswo nicht erbohrten Torflinsen aus irgendeinem Teil der Schichtfolge, was aber nicht sehr wahrscheinlich ist, oder der Fluß hat im Oberlauf ältere Torflager aufgearbeitet und hier sedimentiert. Je nachdem ist entweder die ganze Schichtfolge hier sedimentiert. Je nachdem ist entweder die ganze Schichtfolge über dem Schluffband jünger oder nur ein Teil. Nur im erstgenannten Falle kann über das Alter des Fundlagers verbindlich ausgesagt werden. Nach der ${ }^{14} \mathrm{C}-$ Bestimmung ( ${ }^{14} \mathrm{C}$-Labor des Niedersächsischen Landesamtes für Bodenforschung, Hannover, Dr. GEYH, HV 1952 vom 18. 1. 1968) ergibt sich für den Torf ein Alter von 37150 \pm 1260 Jahre. Der pollenanalytische Befund ergab: „BP: Pinus, Betula, Picea, NBP: Gramineen und Cyperaceen, Interstadial älter als Bölling “ (Dr. H. MülLER, Bundesanstalt für Bodenforschung). Entspricht der Torf dem Schluffband, so muß das Lager von M. eversmanni jünger als 37000 Jahre sein.

Da zu dieser Zeit die Leine unterhalb von Hannover nicht ihren heutigen Verlauf nahm, sondern durch die Wietzeniederung nach Norden abfloß, es aber nicht bekannt ist, wann die Laufänderung erfolgte - zur Zeit der Mittelterrassenbildung floß sie wie in der Gegenwart nach NW - noch wann der Umbau des hydrographischen Systems zum heutigen Stand stattfand, muß es offen bleiben, welchen Zeitabschnitt der Weichselkaltzeit die Leine-Niederterrasse der Wietzeniederung vertritt. Uber das geologische Mindestalter des Fundes können daher ebenfalls keine Angaben gemacht werden. Da wahrscheinlich der Terrassenkörper zeitlich nicht in das Spätweichsel hineinreicht, ergäbe sich für das Objekt ein Zeitansatz zwischen rund 37000 und rund 14000 Jahren. Allenfalls wäre noch zu erwägen, daß das durch den Schädel belegte Exemplar lange nach Abschluß der Sedimentation in den trockenen Flußsanden einen Bau angelegt hat und in diesem zugrunde gegangen sei. Da der lebende Steppeniltis aber seine Baue fast nur in festem, trockenen Lehmboden anlegt, dürfte diese Möglichkeit ausscheiden.

Die Begleitfauna entspricht in ihrer Zusammensetzung der Tiergesellschaft der Weichselkaltzeit, wie sie aus den Niederterrassen der Flüsse Nordwestdeutschlands von zahlreichen Fundstellen her bekannt ist. Eine genauere Aussage über die zeitliche und klimatische Stellung der Terrassensedimente erlaubt sie aber nicht. Dazu müßten unsere Kenntnisse über den Formbestand der älteren Abschnitte der Weichselkaltzeit in unserer Gegend wesentlich besser als zur Zeit sein. Daß Unterschiede sich bei zunehmender Erforschung des Würm-Kaltzeit-Komplexes ergeben werden, scheint mir mit MusIL ziemlich sicher (Musil 1964, Musil-Valoch 1966). Keinesfalls ergeben sich aber Widersprüche zu einem angenommenen Alter, das jünger als 37000 Jahre ist. 
Begleitfauna:

Megaloceros giganteus (BlumenB.)

Rangifer tarandus $\mathrm{L}$.

Bos primigenius BoJ.

Bison priscus (BoJ.)

Mammonteus primigenius (BlumenB.)

Coelodonta antiquitatis (BLUMENB.)

Equus caballus sspec. (untermittelgroß)

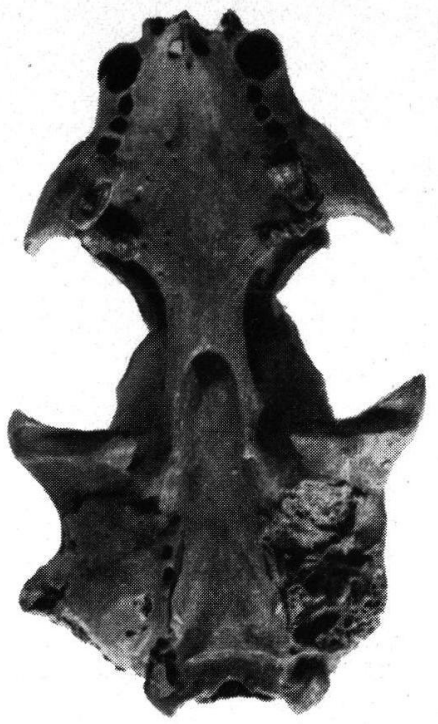

a

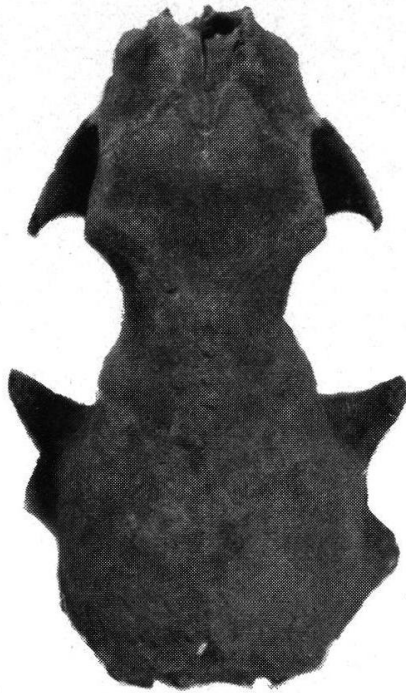

6

Abb. 1. Mustela (Putorius) eversmanni soegeli ÉHIк. Meitze, Niedersachsen. a Schädel, Unterseite, b Schädel, Oberseite. Nat. Gr.

\section{Das Fundstück}

Der Schädel gehörte einem erwachsenen Exemplar an. Er ist ziemlich vollständig, es fehlen nur die beiden Jochbögen, ferner sämtliche Zähne bis auf die beiderseitigen letzten Praemolaren und ersten Molaren. Die Farbe ist gleichmäßig hellbraun, die Zähne sind schwärzlich. Da Abrollungs- oder Scheuerspuren ganz fehlen, darf eine entsprechende Kürze oder überhaupt Mangel eines Transportweges angenommen werden, was allerdings keine Schlüsse über die Weite des Transportweges des Kadavers zuläßt.

Wie aus der Tabelle entnommen werden kann, besteht hinsichtlich der Zugehörigkeit zur Steppeniltis-Gruppe kein Zweifel. Die Breite der interorbitalen Einschnürung ist zwar verhältnismäßig bedeutend, die betreffenden Werte ( 3 und 4 der Tabelle) bleiben aber noch unterhalb der äußersten putorius-Werte. Ebenso entspricht die Form der Nasenöffnung den bei M.eversmanni anzutreffenden Verhältnissen (11-13 der Tabelle). Auch in weniger entscheidenden Merkmalen erweist sich die Zugehörigkeit als sicher, so z. B. in der Länge der Maxillarzahnreihe (Expl. Meitze: 33,2\% der Basilarlänge, $M$. putorius 29.0-32.9) und in der geringen Größe des $M^{1}$ (Breite des $M^{1}$ in $\%$ der Länge der Maxillarzahnreihe, Meitze: $26.8 \%$, M. eversmanni $26.5-28.4 \mathrm{~m}$ in einem Extremwert 
32.0, M. putorius 29.5-34.4\%). Die von den älteren Autoren angegebenen Unterschiede werden von HEPTNER (1964) noch ergänzt: M.eversmanni: Mastoidbreite größer als $1 / 2$ Condylobasallänge, Jochbogenbreite größer als Mastoidbreite, Pterygoidfortsätze nicht oder kaum nach außen gebogen. Das Exemplar Meitze erweist sich auch hierin als eindeutig zu M. eversmanni gehörig. Bestehen also in dieser Hinsicht keine $Z$ weifel an der Frage der artlichen Zugehörigkeit, so ist die Entscheidung nicht leicht zu treffen, ob das Stück ein weiblicher oder männlicher Schädel ist. ZAPFE glaubt ebenso wie seinerzeit HeNSEL deutliche Zeichen für sexuellen Dimorphismus feststellen zu können (1966). Neben der bedeutenderen Größe wäre der männliche Schädel durch weiter ausladende Jochbögen und durch die bedeutendere Hinterhauptsbreite gekennzeichnet. Soweit eine Beurteilung bei der Beschädigung gerade dieser Teile überhaupt möglich ist, so scheint beim Exemplar Meitze das Hinterhaupt recht breit auszuladen $(40 \mathrm{~mm})$, die Jochbögen dürften hingegen nicht sehr stark ausgebuchtet gewesen sein, auch ist die Gesamtgröße eben nicht sehr bedeutend. Wahrscheinlich, aber nicht sicher, war das Exemplar Meitze ein Männchen.

Während heute außerhalb Rußlands der Steppeniltis nur als Unterart von $M$. (P.) putorius angesehen wird (z. B. Ellermann und Morrison-Scott 1951) tritt HeptNer sozusagen als Sprecher der russischen Forscher in einer vor kurzem erschienenen Arbeit (1964) mit, wie mir scheint, guten Gründen morphologischer, ökologischer und zoogeographischer Natur für die Beibehaltung der artlichen Selbständigkeit von $M$.(P.) eversmanni ein. Für die aus dem Auftreten des Steppeniltis in Meitze zu ziehenden klimatischen Schlüsse sind diese Probleme taxionomischer Art ohnehin von untergeordneter Bedeutung. Trotzdem scheint es angebracht, in aller Kürze der Frage nachzugehen, ob der pleistozänen Form West- und Mitteleuropas der Rang einer selbständigen Unterart zuzugestehen sei. Unterschiede zum rezenten osteuropäischen bzw. ungarischen Steppeniltis glaubt schon Soergel 1917 feststellen zu können, er billigt dem pleistozänen Vertreter unterartliche Selbständigkeit zu, verzichtet aber auf eine Namensgebung. Dies besorgt erst ÉHIK 1928, der die Form M.(P.) eversmanni soergeli benennt (N. B. Putorius godoni Duвors 1919 ist nomen nudum, teste Кову 1951). Wenn die artliche Selbständigkeit von $M$. eversmanni bezweifelt wird und man der pleistozänen Form dennoch eine gewisse Sonderstellung einräumen möchte und nicht in „M.(P.) putorius eversmanni LEss." aufgehen läßt, so bliebe als mögliche Benennung nur $M$.(P.) putorius soergeli übrig. Nach SOERGEL, ÉHIK u. a. weichen die mitteleuropäischen Steppeniltisse durch ihre bedeutendere Größe und durch die Zweiwurzeligkeit des P2 von den modernen Vertretern des Formenkreises ab. Was die Größe angeht, so weist Heptner aber darauf hin, daß die in Westsibirien lebenden Angehörigen „Rieseniltisse“ seien. Das Primitivmerkmal „Zweiwurzeligkeit des P2“ tritt beim Eiszeit-Steppeniltis zwar sehr häufig, aber nicht durchgehend auf. Auch bei dem Exemplar Meitze war der $\mathrm{P}^{2}$ nur einwurzelig. Umgekehrt ist auch bei $40 \%$ der rezenten ungarischen Steppeniltisse der P2 (noch ?) zweiwurzelig. Dieses Merkmal rechtfertigt also nicht die Abtrennung einer selbständigen pleistozänen Unterart. Über die Stellung der pleistozänen Form kann daher endgültig erst entschieden werden, wenn Vergleiche auch mit den rezenten asiatischen Verwandten angestellt wurden, da es nicht unmöglich ist, daß jene mit den westsibirischen Iltissen ident ist. $\mathrm{Da}$ aber immerhin eine gewisse Einheitlichkeit der pleistozänen Populationen gegeben erscheint, sollte wenigstens vorläufig die Bezeichnung M.(P.) eversmanni soergeli ÉHIK beibehalten werden.

\section{Zeitliches Auftreten in Mittel- und Westeuropa}

Eine befriedigende Antwort auf die Frage nach dem zeitlichen Auftreten von M.(P.) eversmanni soergeli ÉHIK kann derzeit nicht gefunden werden. Die Einstufungen, soweit sie in der älteren Literatur vorgenommen wurden, entsprechen nicht unserem heutigen Kenntnisstande von der Feinstratigraphie des Würm. Ausdeutungen älterer Berichte sind aber bedenklich, wenn nicht unmöglich. Dazu kommen aber noch Schwierigkeiten grund- 
Maßtabelle

Tabelle 1

\begin{tabular}{|c|c|c|c|c|c|}
\hline & & \multicolumn{3}{|c|}{ M. eversmanni soergeli ÉHIк } & \multirow{3}{*}{$\begin{array}{c}\text { M. putorius (rez.) } \\
\\
\text { n. SoERGEL } 1917 \\
\text { bzw. HENSEL } 1887 \\
\text { n. SoERGEL } 1917\end{array}$} \\
\hline & & \multirow[t]{2}{*}{ Meitze } & \multicolumn{2}{|c|}{ Fuchslucken } & \\
\hline & & & $\begin{array}{c}\hat{\delta} \\
\text { n } 3 \\
\text { n. ZAPFE } 1966\end{array}$ & $\left|\begin{array}{c}q \\
\text { n } 2 \text { (3) } \\
\text { n. ZAPFE 1966 }\end{array}\right|$ & \\
\hline 1 & $\begin{array}{l}\text { Basilarlänge (VRand F.magn.- } \\
\text { HRand mittl. I-Alv.) }\end{array}$ & 62.5 & $\begin{array}{l}63.3- \\
66.2\end{array}$ & $\begin{array}{l}58.0- \\
60.0\end{array}$ & $\begin{array}{l}57.0- \\
66.3 \\
\text { n } 89\end{array}$ \\
\hline 2 & Condylobasallänge & 65.5 & $\begin{array}{l}68.0- \\
71.0\end{array}$ & $\begin{array}{l}62.0- \\
65.0\end{array}$ & - \\
\hline 3 & Br. d. postorb. Einschnürung & 14.1 & $\begin{array}{l}12.8- \\
14.4\end{array}$ & $\begin{array}{l}11.5- \\
13.0\end{array}$ & $\begin{array}{l}14.4- \\
18.2 \\
\text { n } 88\end{array}$ \\
\hline 4 & 4 in $\%$ von 1 & 22.5 & $\begin{array}{l}18.3- \\
21.1\end{array}$ & $\begin{array}{l}18.5- \\
18.7\end{array}$ & $\begin{array}{l}23.9- \\
31.2\end{array}$ \\
\hline 5 & $\begin{array}{l}\text { L. d. Gaumes (HRand mittl. } \\
\text { I-Alv. - Mitte Choanenrand }\end{array}$ & 31.4 & $\begin{array}{l}32.0- \\
33.7\end{array}$ & $\begin{array}{l}30.0- \\
40.0\end{array}$ & $\begin{array}{l}24.3- \\
33.1 \\
\text { n } 89\end{array}$ \\
\hline 6 & $\begin{array}{l}\text { L. VRand C-Alveole - } \\
\text { HRand M1-Alveole }\end{array}$ & 20.7 & $\begin{array}{l}20.5- \\
21.1\end{array}$ & $\begin{array}{l}19.2- \\
19.6\end{array}$ & - \\
\hline 7 & K1. Br. d. Pal. & 7.3 & $\begin{array}{l}7.7- \\
8.7\end{array}$ & $\begin{array}{l}6.7- \\
7.2\end{array}$ & $\begin{array}{l}7.0- \\
8.4 \\
\text { n } 12\end{array}$ \\
\hline 8 & Br. über URand For. infraorb. & 19.4 & $\begin{array}{l}20.5- \\
21.2\end{array}$ & $\begin{array}{l}18.0- \\
18.7\end{array}$ & $\begin{array}{l}18.8- \\
23.3 \\
\mathrm{n} 12\end{array}$ \\
\hline 9 & Br. über ARand d. M1-Alv. & 22.5 & $\begin{array}{l}25.5- \\
25.8\end{array}$ & $\begin{array}{l}21.5- \\
22.7\end{array}$ & $\begin{array}{l}22.6- \\
25.1 \\
\mathrm{n} 6\end{array}$ \\
\hline 10 & $\begin{array}{l}\text { Br. über P4-Alveole } \\
\text { (vorn-außen) }\end{array}$ & 18.3 & $\begin{array}{l}21.0- \\
21.3\end{array}$ & $\begin{array}{l}18.3- \\
20.0\end{array}$ & - \\
\hline 11 & Gr. H. d. Nasenöffnung & 7.5 & $\begin{array}{l}7.7- \\
8.2\end{array}$ & $\begin{array}{l}6.8- \\
7.0\end{array}$ & $\begin{array}{l}8.0- \\
10.0 \\
\mathrm{n} 12\end{array}$ \\
\hline 12 & Gr. Br. d. Nasenöffnung & 8.6 & $\begin{array}{l}8.4- \\
9.0\end{array}$ & $\begin{array}{l}7.0- \\
8.2\end{array}$ & $\begin{array}{l}7.6- \\
8.6 \\
\mathrm{n} 12\end{array}$ \\
\hline 13 & 11 in $\%$ von 12 & 91.0 & $\begin{array}{r}91.1- \\
.2\end{array}$ & $\begin{array}{l}85.3- \\
97.1\end{array}$ & $\begin{array}{l}102.6- \\
120.6\end{array}$ \\
\hline 14 & L. P4 & 7.2 & 一 & - & - \\
\hline 15 & Br. M1 & 5.6 & - & - & - \\
\hline
\end{tabular}


sätzlicher Art, welche der genauen Datierung von Funden aus Lössen und Höhlensedimenten entgegenstehen (s. u.).

Rohdenburg (briefl. Mitt.) hat in der Lößserie des Biedensteg-Aufschlusses (s.u.) einen interglazialen Boden gefunden. Demnach könnte mit einer schon rißzeitlichen Anwesenheit des Steppeniltis in Mitteleuropa gerechnet werden. Eine solche ist aber bislang nicht nachgewiesen, sei es, daß das Fehlen des Steppeniltis auf ihm nicht zusagende Umweltsbedingungen zurückzuführen ist, sei es, daß nur Erhaltungslücken vorliegen. Auch anderen Orts sind präwürmzeitliche Funde unbekannt, obwohl die Art seit langem in Osteuropa und Asien gelebt haben muß.

Der älteste Beleg stammt aus dem Oberen Travertin I des Profiles Ehringsdorf und ist daher in das ausgehende Eem oder in ein sehr frühes Würm einzustufen (KAHLKE 1957/ 58, Guenther 1958). Weitere Altersangaben (auswahlsweise) lauten auf:

„Mousterien“: Höhle b. Liesberg (Кову 1951)

„Altwürm“: Sirgenstein (SOERGEL 1917)

"Aurignacien-Magdalenien“: Kohlerhöhle im Kaltenbrunnertal

(Кову 1951)

„Spätaurignacien-Frühmagdalenien “: Nikolaushöhle bei Veringenstadt

(Hohenzollern) (HeLler 1936)

„Magdalenien“: Weinberghöhlen bei Mauern (Heller 1955)

„Hochmagda!enien“: Hohlefels bei Hütten (STEHLin 1933).

Obwohl die Altersdatierungen durch die älteren Autoren vom heutigen Standpunkt aus nur als allgemeine Hinweise zu gelten haben, kann doch nicht bezweifelt werden, daß die Anwesenheit des Steppeniltis für verschiedene Zeitabschnitte des Würm gesichert erscheint. Es fällt auf, daß für keinen Fund spätmagdalenes Alter angegeben wird. Auch aus klimatologischen Erwägungen ist sein Rückzug aus den westlichen Teilen seines europäischen Areals zu diesem Zeitpunkt ziemlich wahrscheinlich. Reliktvorkommen in Süddeutschland auch im späten Würm wären nicht unmöglich (Mauern [?], HeLler 1955). Nicht zu entscheiden ist beim derzeitigen Kenntnisstande allerdings, ob M. eversmanni zu wiederholten Malen aus seinen östlichen Stammgebieten während ihm zusagender klimatischer Phasen nach Mittel- und Westeuropa zuwanderte oder ob er, wenn zeitweise auch auf Teilareale beschränkt, während des ganzen Würms, sieht man von dessen Endabschnitte ab, hier gelebt hat, was nicht unwahrscheinlich sein dürfte (s. u.).

Als Ergebnis kann festgehalten werden:

a. Gesicherte präwürmzeitliche Vorkommen sind bislang nicht bekannt. Bis auf weiteres hat daher M.eversmanni als zeitliche Leitform des Würm im westlichen Mittel- und Westeuropa zu gelten. Im Spätwürm scheint er sich aber aus diesen Gebieten zurückzuziehen, ob auch aus den östlichen Teilen Mitteleuropas sei dahingestellt (vgl. die gegensätzlichen Auffassungen von ÉHIK (1928) und HePtNer (1964).

b. Da M.eversmanni zumindest im westlichen Mitteleuropa und Westeuropa zu wiederholten Malen während des Würm erscheint, sofern er nicht überhaupt in Teilarealen dauerndes Faunenelement ist, eignet er sich nicht, ältere und jüngere Abschnitte des Würm zu unterscheiden.

\section{Die klimageschichtliche Bedeutung und die ökologischen Ansprüche in Gegenwart und Vergangenheit}

Die Eignung von fossilen Säugetieren zur Ermittlung klimatischer Verhältnisse braucht im einzelnen nicht dargelegt zu werden, obwohl gerade in den letzten Jahrzehnten in dieser Hinsicht Zweifel geäußert wurden oder zumindest ihren Resten nicht die Beachtung geschenkt wurde, die ihnen zukommt. Auf die grundsätzlichen, auch heute noch gültigen Darlegungen von NeHring 1890, Soergel 1919, 1940 zuletzt auch von E. JA- 
COBSHAGEN (1963) darf verwiesen werden. Auf die besonderen Schwierigkeiten, die sich einer Ermittlung der klimatischen Zustände gerade bei den wichtigsten Sedimenten des jüngeren Quartärs, den Lössen und Höhlenablagerungen entgegenstellen, muß allerdings mit allem Nachdruck hingewiesen werden. Was die Lösse angeht, so waren gerade während den Hauptakkumulationsphasen die betreffenden Biotope von einer Vielzahl von Steppensäugern bewohnt, die subterran lebten, entweder dauernd oder doch während der ungünstigen Jahreszeiten bzw. des nachts, und dabei durch ihre Grab- und Wühltätigkeit das ungestörte Sedimentwachstum stark beeinträchtigten, vor allem aber die Skeletteile bereits fossil gewordener Organismen dislozierten (vgl. Tab. S. $X$ ). Viele der in Wohnbauten lebenden Säuger werden in diesen auch zugrunde gegangen sein. Da sie imstande sind, bis zu drei Meter tiefe Baue anzulegen (Marmota bobak MüLL.) wird sich in diesem Falle das Fossillager erheblich tiefer befinden, als dem damaligen Lebensbereich auf der Oberfläche entspricht. Wenn fossile Bodenbildungen knochenführend sind (ein gleiches gilt im übrigen auch von Gastropoden), so liegt der Verdacht nahe, daß darin keine zeitlichen und ökologischen Bezüge zum Boden zum Ausdruck kommen, sondern daß nach Abschluß der Bodenbildung, vielleicht sogar zu einem erheblich späteren Zeitpunkt, diese nur den Untertage-Lebensraum der Subterrannen abgegeben hat, besonders wenn bedacht wird, $\mathrm{daß}$ einer intensiven Bodenbildung rasch die organischen Inhalte karbonatischer Natur zum Opfer fallen werden. Weiter ist zu beachten, daß bekanntermaßen die Löß-Erosion - von Schwemm- und Solifluktions-Lössen ist ohnehin ganz abzusehen - nicht nur von der Oberfläche her angreift, sondern auch intrasedimentär wirksam wird. Kondensationserscheinungen am Fossilinhalt sind daher durchaus möglich. Âhnliches gilt von den Höhlensedimenten. Die Höhlenbewohner irgendwelcher Art einschließlich des Menschen werden durch ihren Aufenthalt unvermeidlich die Schichtfolge spontan und auch nachträglich verändern (vgl. hierzu auch die Ausführungen von Heller 1955). Nicht immer werden daher Fossillager und umgebendes Sediment bei diesen Bildungen eine synchrone Einheit bilden, wobei im Einzelfalle oft gar nicht die Möglichkeit bestehen wird, die zeitlichen Beziehungen eindeutig $\mathrm{zu}$ klären. Feinstratigraphische Untersuchungen können daher unter diesen Voraussetzungen nur mit größter Vorsicht angestellt werden. Nicht unähnlich ist im übrigen die Lage auch bei fluviatilen Ablagerungen mit ihren mannigfachen Umlagerungsvorgängen, allerdings meist quasisynchroner Art. Auf der anderen Seite sprechen aber bestimmte Erfahrungen dafür, daß sich sowohl in Lössen wie in Höhlen gewisse, zeitliche und ökologische Faunenkomplexe ausgliedern lassen, die den Eindruck einer verhältnismäßigen Einheitlichkeit machen (s. Modell 3, S. X). Vielfach wird aber gerade von ökologischer Seite her die Notwendigkeit empfunden werden, die im ganzen gesehen doch groben Komplexe in ökologische und damit vielfach auch zeitliche Abfolgen aufzugliedern; die oben dargelegten Verhältnisse setzen dem aber eine natürliche Grenze, die nach den derzeit gegebenen methodischen Bedingungen nicht durchbrochen werden kann.

Das besagt aber nicht, daß Formen, denen an und für sich ein ökologischer Leitwert zukommt, durch diese Umstände entwertet würden. Nur der genaue Zeitpunkt, wann die durch die Leitform angezeigten Klimaverhältnisse geherrscht haben, läßt sich vielfach nicht feststellen. Im schlimmsten Falle bleibt nur die Aussage übrig, zu „irgendeinem“ Zeitpunkt müssen während der Ablagerung eines Sedimentkörpers oder mindestens in engerer zeitlicher Nachbarschaft zu diesem Vorgange Klimabedingungen geherrscht haben, welche den ökologischen Ansprüchen der betreffenden Art oder Unterart genügt haben.

Um das ökologische Verhalten des Steppeniltis festzustellen, eröffnen sich die auch sonst üblichen Wege, nämlich Beobachtung der gegenwärtigen Lebensweise sowie die Ermittlung der lithofaziellen und biofaziellen „Umwelt" bei Fossilvorkommen. Ergeben sich auffallende Verschiedenheiten zwischen den beiden Befunden, dann erscheint der 
Verdacht begründet, die fossile Form stelle auch bei artlicher Identität eine aus der Gegenwart unbekannte Unterart oder Rasse mit einem abweichenden ökologischen Verhalten dar.

Die biologischen Daten der Steppeniltis-Gruppe (Unterarten von M. eversmanni) sind nach OGNEw (1959) und HePTNER (1964):

Biotope: Halb- und Vollsteppe, Wüste, Hochgebirge, vereinzelt südliche Taiga (offene Stellen).

Geographische Breite: $28^{\circ}$ bis etwas über $60^{\circ}$ (s. Abb. 2), daher nicht in Kontakt mit der hocharktischen Gruppe. Teilkontakte mit der südlichen subarktischen Gruppe (über die ökologischen Gruppen s. S. X).

Wohnung: Unterirdische Bauten in Verbindung mit Vorratshaltung.

Nahrung: Stenophag carnivor, bevorzugt Angehörige der Gattung Citellus, daneben Hamster, Springmäuse und echte Mäuse.

Arealausweitungsverhalten: Nach HePtNER sind die asiatischen Areale ziemlich stabil, anscheinend mit einer leichten Tendenz aus dem Steppengürtel in die nördliche Waldsteppe einzudringen. Die Steppenpopulationen Osteuropas weiten dagegen ihr Areal in der jüngsten Gegenwart fast explosionsartig nach $\mathrm{N}$ und $\mathrm{NO}$ aus, dort offenbar in die neu entstehende Kultursteppe eindringend. Von 1930-1956 wurde die Nordgrenze in Karelien etwa $300 \mathrm{~km}$ weit nach $\mathrm{N}$ vorgeschoben, was einer jährlichen Arealausweitung von durchschnittlich $12 \mathrm{~km}$ entspricht.

Die Lebensansprüche sind also: Winterkaltes, aber sommerwarmes bis -heißes Klima außerhalb des Dauerfrostbodengebietes (eigene Wohnbauten, subterrane Lebensweise der Hauptbeutetiere, s. Tabelle S. $\times$ ), offene Landschaften, Vorhandensein von zahlreichen Nagetieren, insbesondere von Zieselarten.

Die ökologische Valenz ist also gering, was ihn zu einer guten Leitform macht.

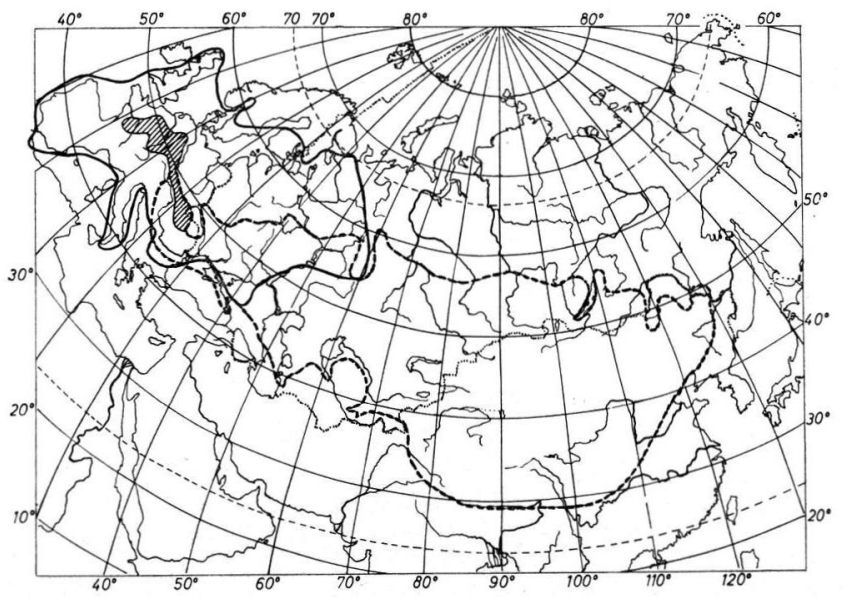

Abb. 2. Die Areale von M.(P.) putorius (ununterbrochene Linie) und M.(P.) eversmanni (gestrichelt); Konturen des Areals von M.(P.) putorius außerhalb der UdSSR mit Korrekturen nach van DER BRINK (1958), nach HeptNer 1964. Das Mindestareal von M.(P.) eversmanni soergeli (schraffiert) wurde auf Grund der vorliegenden Fundortsangaben in die Karte von HEPTNER durch den Autor eingetragen.

Das ökologische Verhalten von M. eversmanni im europäischen Würm wurde an Hand von drei „Modellen“ zu überprüfen versucht. Da aus den oben dargelegten Gründen der Sedimentbeschaffenheit dabei nur eine zweitrangige Bedeutung zukommt, bildet daher die ökologische Analyse der „Begleitfauna“ bzw. der faunistischen Abfolge die Hauptstütze. 
Die Säugetiere wurden fünf verschiedenen Gruppen zugeteilt, und zwar:

\author{
Steppengruppe \\ Mitteleuropäische Gruppe \\ Subarktische Gruppe \\ Hocharktische Gruppe \\ Gruppe von unsicherem ökologischen Charakter.
}

Im einzelnen ist dazu folgendes zu bemerken:

Steppengruppe: Umfaßt die Bewohner der heutigen osteuropäischen und asiatischen, nördlichen semiariden und ariden Gebiete ohne Aufgliederung in eine "wärmere" und „kältere“ Fazies. Kleinsäuger hauptsächlich grabend und wühlend (s. Tab. S. X).

Mitteleuropäische Gruppe: Aus der Gegenwart aus Mitteleuropa bekannt. Der ökologische Leitwert ist verhältnismäßig gering, da es sich meist um Arten mit einem sehr großen Areal handelt. Von Wichtigkeit wäre die Identifizierungsmöglichkeit mit den jeweiligen heutigen Unterarten, die in der Regel ein bestimmtes ökologisches Verhalten zeigen. Nur an Hand von osteologischem Material ist dies aber nur selten möglich. Im ganzen gesehen spricht aber eine Dominanz dieser Gruppe gegen das Bestehen strenger und einseitiger Klimabedingungen.

Subarktische Gruppe: Dazu wurden die Bewohner des heutigen nördlichen Waldgürtels und der südlichen Tundrengebiete gezählt.

Hocharktische Gruppe: Heute ausschließlich auf die baumfreien, nördlichen Tundrengebiete beschränkt. Keine Grab- und Wühlformen (s. Tab. S. $\times$ ).

Gruppe von unsicherem ökologischen Charakter: Dazu gehören sämtliche ausgestorbenen Formen, deren Ökologie nur auf indirektem Wege, behaftet mit Unsicherheitsfaktoren, erschlossen werden kann. Bei den untersuchten Modellen haben wir es zwar ausschließlich mit den Großsäugern der „Kaltzeiten“ zu tun. Da trotz scheinbar guter Kenntnis der in Betracht kommenden Formen deren ökologische Ansprüche - offenbar von Unterart (Ókorasse) zu Unterart etwas verschieden - im einzelnen noch mehr oder weniger unbekannt sind, kommt ihrer Anwesenheit meist nur der Erkenntniswert „glaziale“ oder „interglaziale“ Temperaturverhältnisse zu. Wenigstens derzeit kann in der Regel nicht auf „Steppe“, „Steppenwald“, „subarktische oder hocharktische Tundra“ usw. entschieden werden.

Gestein und

Modell 1: Fuchslucken (N.Oestr.) (EHrenberg 1966)

Schichtmächtigkeit: sandig-schluffige Höhlensedimente, über $2 \mathrm{~m}$

Paläontologische

(säugetierpaläonto-

logische) Datierung: Sicheres Würm, wahrscheinlich mittlere Abschnitte, Spätwürm kann ausgeschlossen werden

Prähistorische

Datierung:

Horizontierung:

wahrscheinlich Aurignacien, Szeletien oder Gravettien, kaum Magdalenien Höhle hauptsächlich besiedelt durch Höhlenhyäne und -bär, daneben eingangsnahe auch durch kleinere Raubtiere (Fuchs, Dachs, Iltis), daher gesicherte Aufgliederung in einzelne Horizonte nicht möglich

OKologische Gruppen:

\footnotetext{
Steppengruppe:

10 Arten (Mustela eversmanni soergeli ÉHI 1 ), Lepus euro p a e us PALL., Ochotona pusilla fossilis GeRnv., C i t e l l us cit e ll $\mathrm{l}$ s ci t e ll o id es Korm., Allactaga cf. major KerR, Cricetus cricetus L., Clethrionomys glareolus istericus Mill., Microtus (Stenocranius) gregalis PALl., Equus (Equus) cfr. chosaricus Gromova.

Subarktische bis (hocharktische) Gruppe:

6 Arten (Alopex lagopus L., Gulo gulo L., Lepus timidus L., Sicista betulina Pall., Microtus oeconomus ratticeps KaYs. u. BLAs., $R$ a $n g$ if er $t$ a $r$ a $n-$ $d u s \mathrm{~L}$.
}

1) Vorherrschende Arten sind durch Sperrung gekennzeichnet. 
Okologische Gruppen:

Gestein und

Schichtmächtigkeit: einige Meter Löß

Säugetierpaläonto-

logische Datierung: Sicheres Würm

Horizontierung: Da Bodenwühler vorhanden sind, ist eine sichere Horizontierbarkeit nicht

Ökologische

Gruppen:

Hocharktische Gruppe:

Lemmus lemmus L. (Anwesenheit fraglich), Dicrostonyx benseli HiNT. (sehr selten)

mitteleuropäische Gruppe:

11 Arten (Canis lupus L., Vulpes vulpes L., Meles meles L., Mustela cfr. erminea L., M. nivalis L., Martes cfr. martes L., Talpa europaea L., Castor fiber L., Arvicola terrestris scherman SHAw, C. elapbus sspec., Bison bonasus L. (Anwesenheit fraglich), Microtus arvalisa grestis - Gruppe)

ökologischer Leitwert unsicher, vermutlich bes.

Waldsteppe und Steppe und Tundra (?):

8 Arten (Crocuta spelaea (Goldf), Ursus spelaeus RoSEnM., Panthera spelaea (Goldf.), Me galoceros giganteus (BLUMENB.), Bison priscus (BoJ.), Mammonteus primigenius (BLUmenb.), Coelodonta antiquitatis Blumenb., Equus (? Asinus) hidruntinus REG.

Deutung:

Wechsel und/oder Verzahnung von Halbsteppe (Seltenheit bzw. Fehlen der Charakterformen der Vollsteppe, sämtliche dominante Arten kommen auch heute noch im Gebiet vor) und Wald bzw. Steppenwald mit wenigen, schwachen Vorstößen der subarktischen und - kaum angedeutet - der hocharktischen Gruppe; „wärmste“ Variante der drei Modelle.

Modell 2: Mauer bei Heidelberg (Oberrheintalgebiet) (Wurm 1913) möglich.

Steppengruppe:

3 Arten $\left.{ }^{2}\right)$ (M.[P.] eversmanni soergeli ÉHIK, Citellus major PALl., Lepus europaeus L.)

subarktische Gruppe:

2 Arten (Microtus oeconomus ratticeps Kays. u. Blas., Rangifer tarandus L.) mitteleuropäische Gruppe:

4 Arten (Talpa europaea L., Arvicola terrestris L., Microtus arvalis Pall., Vulpes vulpes L.)

Unsicher: Equus spec.

Deutung:

Sieht man von dem wesentlich ärmeren Formenbestand $a b$, so entspricht Mauer ökologisch der Fuchsluckenfauna, d. h. Wechsel und/oder Verzahnung von Halbsteppe (s. Modell 1) und Wald bzw. Steppenwald mit schwachen Vorstößen der sub- und hocharktischen Gruppe.

Modell 3: Lehmgrube „Biedensteg“ bei Bad Wildungen (N.Hessen)

Gestein und

(Jacobshagen, E., Huckriede, R., Jacobshagen, V. 1963)

Schichtmächtigkeit: Lösse mit Fließerden und interwürmzeitlicher Bodenbildung; $6 \mathrm{~m}$

Geologische

Datierung:

Altwürm-Paudorfer Bodenbildung - Jungwürm;

ROHDENBURG erwägt eine interglaziale Bodenbildung, danach wären die liegenden Lösse rißzeitlich (briefliche Mitteilung). Die Altersfrage ist für unsere Fragestellung von untergeordneter Bedeutung; sollten die liegenden Lösse rißzeitlich sein, so würde ihre Steppenfaunen eine der ältesten ihrer Art in Mittel- und Westeuropa sein; zugleich wäre es auch der älteste zeitliche Beleg für M.(P.) eversmanni.

Säugetierpaläontologische Datierung:

Wie in der obengenannten Arbeit ausgeführt, sind Artbestand und -vergesellschaftung für würmzeitliche Sedimente charakteristisch. Dem kann voll

2) Die Benennung der Arten folgt dem derzeitigen Stand der Nomenklatur. 
Ókologische Gruppen: beigepflichtet werden. Es muß allerdings bedacht werden, daß Steppenfaunen aus dem Riß kein wesentlich anderes Bild bieten würden. Andererseits darf der Hinweis auch nicht unterbleiben, daß von säugetierpaläontologischer Seite kein hinreichender Grund besteht, die liegenden Lösse ins Altwürm einzustufen, wozu ausreichendes Material von Großsäugern vorhanden sein müßte. Nur mit Hilfe der Kleinsäuger läßt sich, wenigstens zur Zeit, die Frage nicht entscheiden. Es genügt in diesem Zusammenhang die generelle Feststellung „höchstwahrscheinlich Würm“. Die Faunenführung beschränkt sich im wesentlichen auf den älteren Lößkomplex, aus der jüngeren Lößabfolge liegen nur sehr dürftige Reste einer Tundren(?)-Fauna vor.

Der ältere Lößkomplex kann in vier Horizonte aufgegliedert werden. Nach Anzahl der Reste erlaubt nur der Horizont III eine statistische Auswertung. Bei einer Häufigkeit von grabenden Formen ist die genaue Horizontierbarkeit im einzelnen problematisch. Murmeltierbauten scheinen nachweisbar.

Horiz on t I : 1.7-2.4 m Tiefe im Gesamtprofil, im Hangenden des älteren Lößkomplexes unterhalb der Bodenbildung.

Steppengruppe:

5 Arten $(M .[P$.$] eversmanni soergeli ÉHIK, Citellus major PALl., Allactaga$ major KerR, Marmota bobak ? MüLL., Ochotona pusilla PALL.)

Mitteleuropäische Gruppe:

4 Arten (Vulpes vulpes L., Meles meles L., Sus scrofa L., Cervus elaphus sspec. (sub C. spec.)

unsicher: 3 Arten (Crocuta spelaea (GoLDF.), Megaloceros giganteus (BLuMENB.), sub Cervus megaceros germaniae PoHL.), Equns caballus sspec. (sub E. germanicus NeHR.)

Deutung:

Wärmere Vollsteppe, später übergehend in Wald (Bodenbildung!), keine arktischen Einstrahlungen.

Horizon t I : $2.4-3.0 \mathrm{~m}$ Tiefe

Subarktische Gruppe:

3 Arten (Alopex lagopus L., Ursus arctos L., Rangifer tarandus L.)

Unsicher: 2 Arten (Equus caballus sspec. (sub E. germanicus Nenr.), Mammonteus spec. (sub Elephas (M.) primigenius Blumenb.)

Deutung:

Grenzgebiet Wald-Tundra

Horizon t I I I : Gewöllschichten) $3.0-3.3 \mathrm{~m}$ Tiefe

Steppengruppe:

5 Arten Microtus (Stenocranius) gregalis Pall., 60.2\% d. Microtini, Phodopus sungorus Pall., Citellus major Pall., Allactaga major Kerr, Ochotona pusilla PALL.)

Subarktische (bis hocharktische) Gruppe:

Microtus nivalis MART., $1.4 \%$, M. oeconomus ratticeps KAYS. u. Blas., $9.8 \%$, Alopex lagopus L.

Hocharktische Gruppe:

2 Arten (Dicrostonyx henseli Hint., $7.5 \%$, Lemmus lemmus L. 0,4\%)

Mitteleuropäische Gruppe:

8 Arten (Sorex araneus L., S. minutus L., Talpa europaea L., Canis lupus L., Mustela vulgaris EnxL., Arvicola terrestris L., Clethrionomys glareolus 4.6\% Microtus agrestis-arvalis-Gruppe, $10.1 \%$

Unsicher: 2 Arten (Lepus spec., Microtus brandi BrunN. (zweifelhafte Art)

Deutung:

kalte Steppe (Dominanz von M. gregalis) in Biotopverzahnung mit Tundra, Feuchtstellen und begünstigteren Standorten, Grenzen oszillierend, vermutlich nach unten übergehend in wärmere Steppe, kälteste, kurzwährende Phase der Abfolge

Horizont IV: $3.3-6.0 \mathrm{~m}$ Tiefe

Steppengruppe:

7 Arten (M.[P.] eversmanni soergeli ÉHIK ? (sub P. putorius L., da das Objekt aber spezifisch nicht bestimmbar ist, eher M. eversmanni als $M$. putorius), Ochotona pusilla PALl., Citellus major PALl., Allactaga major KerR, Marmota spec., Phodopus sungorus PaLl., Microtus gregalis PalL.; außerdem Murmeltierbauten 
Ơkologische Gruppen:

Subarktische Gruppe:

3 Arten (Alopex lagopus L., Microtus oeconomus ratticeps Kays. u. Blas., Rangifer tarandus $\mathrm{L}$.

Hocharktische Gruppe:

2 Arten (Lemmus lemmus L., selten, Dicrostonyx henseli Hint., 1 Exemplar unmittelbar unter dem Horizont III)

mitteleuropäische Gruppe:

9 Arten (Erinaceus europaeus L., Talpa europaea L., Sorex araneus L., S. minutus L., Neomys spec. (sub $N$. fodiens Schreb.), Vulpes vulpes L., Meles meles L., Arvicola terrestris L., Clethrionmys glareolus L., Microtus agrestisarvalis-Gruppe

Unsicher: 10 Arten (Crocuta spelaea (GoldF.), Lepus spec., Microtus brandi Brunn. (s. o.), Cervus elaphus sspec. (sub C. spec.), Megaloceros giganteus Blumenb.) (sub Cervus megaceros germanicus PoHL.), Bison priscus BoJ., Mammontheus primigenius (Blumens.), Coelodonta antiquitatis (Blumenв.) (sub C.lenensis Pall.), Equus caballus sspec. (sub E. germanicus NeHr.), E. hemionus Pall. oder ? Asinus hidruntinus Reg. (sub. E. hemionus Pall.) Deutung:

$\mathrm{Zu}$ oberst Tundra-Kalte Steppe nach unten rasch übergehend in eine BiotopVerzahnung von Steppenwald und wärmerer Vollsteppe mit Feuchtstellen mit stark oszillierenden Grenzen, die Ausdruck kurzfristiger Klimaschwankungen sein dürften.

Aufgrund dieser Modelle können folgende Vorstellungen über die Umweltsansprüche von M. eversmanni soergeli erhoben werden:

a. Der fossile Steppeniltis ist keine ökologische Sonderform, da er in Gesellschaft von anderen Steppentieren auftritt. Bezeichnenderweise wird er in allen Fällen von Zieselarten begleitet, die offenbar ebenso wie in der Gegenwart auch in der Vergangenheit seine Hauptnahrung bildeten. Dies gilt im übrigen auch für die überwiegende Mehrzahl seiner anderen, hier nicht erwähnten pleistozänen Vorkommen. Wie schon Soergel (1919) hervorhebt, ist zwar bei der einen oder anderen Art bei klimatischen Änderungen die Entstehung von neuen Ớkorassen denkbar, daß aber eine gesamte Biozönose sich ökologisch umformt, widerspricht allen Erfahrungen.

b. Solche Umprägungsvorgänge wären allenfalls noch vorstellbar, wenn eine Biozönose durch Umweltsänderungen aus dem gesamten bisherigen Areal verdrängt würde, ohne daß sich Ausweichmöglichkeiten in äquivalente Biotope geboten hätten. Das mag im kaltzeitlichen Eurasien am ehesten noch für die arktische, subarktische und alpine Gruppe zutreffen, die das wachsende Eis vor sich herschob, nicht aber für die östlichen Steppengesellschaften, die keinem solchen Druck ausgesetzt waren.

c. Sind irgendwo Gesellschaften der wärmeren oder kälteren östlichen Steppe in Mittel- und Westeuropa anzutreffen, dann haben Umweltsverhältnisse geherrscht, die auch den heutigen Lebensansprüchen genügten, da anderenfalls die Arealausweitung unverständlich wäre (über die Bedeutung der Arealverschiebungen in ost-westlicher Richtung zuletzt besonders E. JACOBSHAGEN 1963).

d. Die Rekonstruktion der Umwelt kann daher mit an Sicherheit grenzender Wahrscheinlichkeit für M.eversmanni soergeli nach den für die Steppeniltis-Gruppe heute ermittelten Daten vorgenommen werden. Sie lautet: Offene Landschaften (Gräser- und Kräutersteppen) mit mäßigen bis geringen Niederschlägen in winterkalten, aber sommerwarmen Klimaten außerhalb des Dauerfrostbodenbereiches (s. auch Tabelle S. X).

e. Uber die Vegetation der kaltzeitlichen Steppen in Europa liegen Untersuchungen von Frenzel vor $(1964,1967)$. - Vorherrschend waren teils Gräser, teils Kräuter, vor allem Artemisia-Arten, Chenopodiaceen und andere, mehr oder weniger halophile Gewächse. Das ist die gleiche Vegetation, wie sie in den heutigen Hauptverbreitungsbereichen des Steppeniltis vorkommt. Zwischen den paläozoologischen und paläobotanischen $\mathrm{Be}-$ funden bestehen demnach keinerlei Widersprüche. 


\section{Tabelle 2}

Ơkologie der wichtigsten Tundren- und Steppennager und - lagomorphen des mitteleuropäischen Jungpleistozäns in der Gegenwart

\begin{tabular}{|c|c|c|c|c|}
\hline & $\begin{array}{l}\text { Nördl.-südl. } \\
\text { Breite } \\
\text { (Angaben } \\
\text { ungefähr) }\end{array}$ & Landschaft & Nahrung & $\begin{array}{l}\text { Unterirdische } \\
\text { Baute }\end{array}$ \\
\hline \multicolumn{5}{|l|}{ Steppengruppe } \\
\hline Ochotona pusilla PALL. & $\begin{array}{l}55^{\circ}-30^{\circ} \\
\text { (Hochgebirge, } \\
\text { Unterarten!) }\end{array}$ & $\begin{array}{l}\text { Zwergstrauch- } \\
\text { bestände }\end{array}$ & $\begin{array}{l}\text { Kräuter, bes. } \\
\text { Artemisia }\end{array}$ & $\begin{array}{l}\text { Kessel bis } 0.7 \mathrm{~m} \\
\text { Tiefe }\end{array}$ \\
\hline Marmota bobak MüLL. & $\begin{array}{l}53^{\circ}-28^{\circ} \\
\text { (Hochgebirge, } \\
\text { versch. Unter- } \\
\text { arten!) }\end{array}$ & $\begin{array}{l}\text { Steppe, } \\
\text { Hochgebirge }\end{array}$ & $\begin{array}{l}\text { Wurzeln, } \\
\text { Kräuter }\end{array}$ & $\begin{array}{l}\text { Kessel bis über } \\
3 \mathrm{~m}\end{array}$ \\
\hline Citellus citellus $\mathrm{L}$. & $52^{\circ}-40^{\circ}$ & $\begin{array}{l}\text { offene Lehm- } \\
\text { steppe }\end{array}$ & $\begin{array}{l}\text { zarte Kräuter } \\
\text { u. Wurzeln }\end{array}$ & Kessel bis $2 \mathrm{~m}$ \\
\hline $\begin{array}{l}\text { Citellus major PaLl. } \\
\text { (meist sub Colobotis } \\
\text { rufescens KaYs u. BLAS.) }\end{array}$ & $56^{\circ}\left(60^{\circ}\right)-45^{\circ}$ & $\begin{array}{l}\text { offene Lehm- } \\
\text { steppe }\end{array}$ & Gräser, Kräuter & Kessel bis $2 \mathrm{~m}$ \\
\hline $\begin{array}{l}\text { Allactaga major KERR } \\
\text { (meist sub A. saliens GMEL.) }\end{array}$ & $52^{\circ}-45^{\circ}$ & $\begin{array}{l}\text { offene Lehm- } \\
\text { steppe }\end{array}$ & $\begin{array}{l}\text { Monocoty- } \\
\text { ledonen- } \\
\text { Zwiebeln }\end{array}$ & tiefe Kessel \\
\hline $\begin{array}{l}\text { Phodopus sungorus PaLL. } \\
\text { (meist sub Cricetulus phäus } \\
\text { PaLL.) }\end{array}$ & $\begin{array}{l}\text { Südsibirien } \\
\text { Mongolei } \\
\text { Mandschurei }\end{array}$ & offene Steppe & $\begin{array}{l}\text { Samen } \\
\text { (Potentilla, } \\
\text { Stipa, Allium, } \\
\text { Iris usw.), } \\
\text { Insekten, bes. } \\
\text { Käfer }\end{array}$ & $\begin{array}{l}\text { horizontale } \\
\text { Gänge m. } \\
\text { Nestkammer } \\
(0.25-0.30 \mathrm{~m})\end{array}$ \\
\hline $\begin{array}{l}\text { Microtus (Stenocranius) } \\
\text { gregalis PALL. }\end{array}$ & $\begin{array}{l}78^{\circ}-40^{\circ} \\
\text { (Unterarten!) }\end{array}$ & $\begin{array}{l}\text { offene Steppe } \\
\text { (bes. Grassteppe) } \\
\text { und? }\end{array}$ & $\begin{array}{l}\text { alle Steppen- } \\
\text { pflanzen }\end{array}$ & $\begin{array}{l}\text { Tiefe der } \\
\text { Bauten? }\end{array}$ \\
\hline \multicolumn{5}{|l|}{ Subarktische Gruppe } \\
\hline Lepus timidus L. & $80^{\circ}-48^{\circ}$ & $\begin{array}{l}\text { Tundra, Wald, } \\
\text { bewachsene } \\
\text { Moore, Steppe }\end{array}$ & $\begin{array}{l}\text { sehr verschieden- } \\
\text { artig }\end{array}$ & - \\
\hline $\begin{array}{l}\text { Microtus oeconomus } \\
\text { ratticeps KaYs u. BLAS. }\end{array}$ & $70^{\circ}-48^{\circ}$ & $\begin{array}{l}\text { feuchte } \\
\text { Ländereien }\end{array}$ & $\begin{array}{l}\text { Cyperaceen, } \\
\text { Wurzeln, } \\
\text { Kräuter }\end{array}$ & oberflächlich \\
\hline \multicolumn{5}{|l|}{$\begin{array}{l}\text { Hocharktische } \\
\text { Gruppe }\end{array}$} \\
\hline Lemmus lemmus $\mathrm{L}$. & $70^{\circ}-59^{\circ}$ & $\begin{array}{l}\text { Moos- u. } \\
\text { Zwergstrauch- } \\
\text { tundra }\end{array}$ & $\begin{array}{l}\text { bes. Moose, } \\
\text { Gräser, } \\
\text { Flechten, } \\
\text { Wurzeln }\end{array}$ & - \\
\hline $\begin{array}{l}\text { Dicrostonyx torquatus } \\
\text { PALL.-Gruppe } \\
\text { (D. guliemi Hint. } \\
\text { D. henseli HiNT. } \\
\text { im europäischen Pleistozän) }\end{array}$ & $\begin{array}{l}82^{\circ}- \\
\text { Waldgrenze }\end{array}$ & Tundra & $\begin{array}{l}\text { bes. Carex- } \\
\text { Arten, } \\
\text { Eriophorum }\end{array}$ & - \\
\hline
\end{tabular}




\section{Der Steppeniltis in der Klimageschichte des Würm}

Gegenstand der weiteren Überlegungen hat zu sein, in welchen Zeitabschnitten bzw. Klimaphasen innerhalb des mittel- und westeuropäischen Würms die Voraussetzungen für die Anwesenheit des Steppeniltis gegeben waren. Sind diese in den "Interstadialen“ oder „Stadialen“ zu suchen?

Interstadiale: Nach ziemlich übereinstimmender Auffassung war das Klima in wechselndem Ausmaße wärmer als zur Zeit der Stadiale; auch war der Wasserhaushalt für die Vegetation günstiger. Zumindest in den älteren Interstadialen sind daher Waldgesellschaften weit verbreitet gewesen. An mannigfachen paläobotanischen Belegen fehlt es nicht (Frenzel 1967). Dafür sprechen auch bodenkundliche Forschungen an interstadialen Böden, u. a. im südlichen Nordwestdeutschland (Rohdenburg u. MeYer 1966). Dies gilt insbesondere für die Altwürm-Böden; aber auch noch die Bodenbildung der Paudorf-Zeit dürfte in diesen Gebieten unter Waldbedeckung stattgefunden haben. Diese Annahme wird durch unveröffentlichte Untersuchungen über die Fauna und Flora von Schinnal Weser aus der Oberen Niederterrasse der Weser bestätigt. Die Alterswerte umfassen eine Spanne, die von 28000 bis 32000 reicht. Zwischen Paudorf und dem Bölling-Alleröd ist bislang Bewaldung weder botanisch noch pedologisch nachweisbar. Nach dem oben Gesagten geben aber interstadiale Waldlandschaften, auch wenn diese von Waldsteppe oder Steppenwäldern besetzt waren, für den Steppeniltis keine geeignete Biotope ab. Selbst wenn die Gegenwarts-Beobachtungen auf beginnendes Eindringen in die Waldsteppe zutreffen, so ist nicht vorstellbar, daß die ihn begleitenden Elemente (Ziesel, Pferdespringer, Murmeltiere, Zwerghamster) gleichfalls sich zu Waldbewohnern gewandelt hätten (s. o.) (s. Tabelle S. X). Höchstwahrscheinlich werden sich zwar in allen Interstadialen an geeigneten Orten größere oder kleinere Steppeninseln mit entsprechender Fauna und Flora gehalten haben (echte Steppenböden, die einem der bekannten Interstadiale zuzuordnen wären, sind allerdings bislang unbekannt). Sie sind aber nur Restbiotope für Gesellschaften, die sich in die Waldzeit hineingerettet haben, um bei Wiederkehr von für sie günstigerer Klimabedingungen wieder Großareale zu besetzen. Höchst unwahrscheinlich ist es aber, daß in irgendeinem Interstadial Steppeninseln am Nordrand der Mittelgebirge so ausgedehnt gewesen wären, daß sie als Heimat des Exemplares von Meitze in Betracht $\mathrm{zu}$ ziehen sind. Es muß hier vielmehr in einem Stadial gelebt haben.

Stadiale: Nach den bisherigen Überlegungen ist zu fordern, daß wenigstens zeitweise ein Klima geherrscht habe, das in Mittel- und Westeuropa den Säugern der östlichen Steppen Lebensmöglichkeiten geboten habe. Dagegen wird einzuwenden sein, daß

1. überreiche Zeugnisse von Solifluktion, Kryoturbation und anderen Anzeichen des Dauerfrostes nachweisbar sind;

2. Steppenböden fehlen;

3. neben den Steppen- und Steppenwaldformen Arten sub- und hocharktischer Biotope in etwa den gleichen Straten auftreten.

Im einzelnen auf diese durchaus berechtigten Einwände einzugehen, verbietet die notwendige Begrenzung des Umfanges. Trotzdem können sie nicht übergangen werden, sondern erfordern wenigstens Hinweise, wie die Widersprüche sich lösen lassen:

$\mathrm{Zu}$ Punkt 1: Derzeit gibt es keine Mittel, die zeitliche Dauer der ausgesprochenen Periglazialphänomene festzulegen. Gegen die Annahme eines ständigen Wechsels von trokken-,warmen“ und trocken-streng-kalten Phasen lassen sich keine stichhaltigen Beweise erbringen. Gerade die jüngsten bodenkundlichen Untersuchungen in unserem Gebiet zeigen, daß mit einem sehr häufigen, wahrscheinlich abrupten Wechsel von Lößakkumulation und Eiskeilbildung als Merkmal strenger Dauerkälte gerechnet werden muß (ROHDENBURg u. Meyer 1966). 
Zu Punkt 2: Die Annahme eines kalten Dauerklimas während der „Stadiale“ beruht auf Gedankengängen, die sich auf folgende kurze Formel bringen lassen: Interstadiale = Zeit der Bodenbildung, Stadiale = Mangel an Bodenbildung, da zu kalt. Frenzel (1967) weist wohl mit Recht darauf hin, daß Bodenbildungsvorgänge nicht ohne weiteres eine Funktion von "warm" und "kalt" seien, sondern daß in Lössen während der Hauptanwehungszeit eine synchrone Bodenbildung nicht leicht vorstellbar sei. Es ergibt sich daher ebenso ein funktioneller Zusammenhang mit „feucht" und „trocken“. Wenn eine Bodenbildung während der Akkumulationsphasen aus klimatisch-physikalischen Zuständen heraus eine Einschränkung erfahren mußte, so nicht minder auch aus biologischen. Die Beziehungen zwischen den Steppentieren und dem Untergrund, wie sie heute in Zentralasien herrschen, werden von OGNEw aufgrund zahlreicher Untersuchungen russischer Biologen so anschaulich geschildert, daß sie verdienten, im Wortlaut wiedergegeben zu werden (OGNEw 1959, S. 144-153). Danach kann die Tätigkeit vor allem der Subterrannen auf die Gestaltung des Kleinreliefs, auf die Unterbindung der Bodenprofilbildung durch das ständige Durchwühlen des Bodens unter Herausbringen tieferer Schichten (dadurch auch Entstehen einer Salzflora!) und auf die Mobilisation toniger und schluffiger Lockermassen und deren Bereitstellung zum äolischen Transport nicht hoch genug veranschlagt werden. Es können daher auch in Europa gerade in den wärmeren Abschnitten der Stadiale mit ihrem reichen Tierleben nicht deutlich ausgeprägte Böden entstanden sein, so daß deren Fehlen nicht unter allen Umständen den Schluß auf ein „zu kalt“ rechtfertigt.

Zu Punkt 3: Das „Zusammenvorkommen“ von verschiedensten, jeweils an sehr unterschiedliche Klimate angepaßten Formen war schon für die älteren Generationen der Quartärpaläontologen ein Hauptproblem. Man ging allerdings von der, damals unwidersprochenen Voraussetzung von sehr lange währenden, gleichmäßig getönten Klimaperioden während der „Eiszeit“ aus. Das Bild wird aber einfacher, wenn die Ergebnisse der jüngsten Quartärforschung zugrunde gelegt werden, die insgesamt dafür sprechen, daß gerade der Würm-Kaltzeitkomplex eine Zeit sehr häufigen und offenbar recht krassen Klimawechsels war. Daß die Mischfaunen wenigstens teilweise künstlicher Natur sind, konnte bereits gezeigt werden. Offensichtlich hat es aber auch echte gegeben. Schon SoeRgEL (1919) weist aber darauf hin, daß eine wirkliche Biotopverzahnung z. B. von hocharktischen und Steppenbiozönosen nicht als stabiler Zustand gedacht werden kann. Sie können daher nur für die sehr kurzfristig zu bemessenden Zeiten klimatischen Umschwungs bestanden haben. Aus dem gemeinsamen Vorkommen in einer Schicht darf daher nicht geschlossen werden, das ökologische Verhalten der pleistozänen Säuger sei, wie oft vermutet, ein grundsätzlich anderes gewesen als in der Gegenwart. Die Mischfaunen haben vielmehr als deutlicher Hinweis dafür zu gelten, daß sich der Klimawechsel bei anhaltender Tendenz $\mathrm{zu}$ ausgiebigen Klimaschwankungen sehr rasch vollzogen hat. Dabei mußte es einerseits $\mathrm{zu}$ echten Biotopverzahnungen kommen, andererseits spiegelt sich in der Mischung ein zeitliches Nacheinander wider, daß bei der Kürze der Zeiträume mit den derzeitigen Methoden sich nicht in seine einzelnen Phasen auflösen läßt. Daß die Fauna rascher und nachhaltiger solchen klimatischen Vorgängen folgen wird als die Vegetation, darauf haben unlängst Dietz, Grahle und Müller hingewiesen (1958). Was dort für die Mollusken gefordert wird, hat erhöhte Gültigkeit für die noch wesentlich beweglicheren Säuger. In diesem Zusammenhang sei nochmals auf die heute zu beobachtende Ausbreitungsgeschwindigkeit von M.eversmanni in Europa hingewiesen. Selbst Klimaschwankungen von nur 500 Jahren Periodendauer, im Sediment kaum mehr nachweisbar, vermochten eine Arealausweitung von einigen tausend Kilometern herbeizuführen. Wenn das Auftreten von Gesellschaften, die nur in einer nicht extrem kalten, bodenfrostfreien Steppe gelebt haben können, während der Stadiale belegt ist, dann darf diese Aussage weder ökologisch noch klimatisch durch den Hinweis auf die „Mischfauna“ abgewertet werden. 
Die Quartärforschung unserer Tage ist auf dem besten Wege, die Interstadiale in einzelne Phasen von klimatisch beträchtlichem Unterschied aufzulösen (z. B. Brørup-Interstadial, bei Frenzel 1967). Zukünftigen Forschungen wird es vorbehalten sein, ein gleiches mit den Stadialen zu versuchen, wobei die Hauptaufgabe der Säugetierpaläontologie und der am Anfange stehenden Löß-Botanik (Frenzel 1964) zufallen wird. Das Ergebnis läßt sich bereits ahnen: Auch die Stadiale umfassen starke Klimaschwankungen, die von den echten, streng kalten Glazialen und Periglazialen zu den "verborgenen“, durch keine Bodenbildung belegten "Interstadialen“ der ebenso trockenen, aber wahrscheinlich sogar wesentlich wärmeren Phasen reichen.

\section{Literaturverzeichnis}

BRINK van DEN, F. H.: Die Säugetiere Europas westl. d. 30. Längengrades. 225 S., 470 Abb., 30 Taf., Hamburg-Berlin 1956.

Dietz, C., Grahle, H.-O., Müller, H.: Ein spätglaziales Kalkmudde-Vorkommen im Seck-Bruch bei Hannover. Geol. Jb. 76, 67-102, 9 Abb., Hannover 1958.

Dubois, A. \& Stehlin, H. G.: La Grotte de Cotencher, station mousterienne. 178 S., Abh. Schweiz. Pal. Ges. 52, 9 Taf., 27 Abb., Basel 1932/33.

Ériк, J.: Der Steppeniltis (Mustela eversmanni Less.) in Ungarn. Ann. Mus. Nat. Hung. 25, 1-28, Budapest 1928.

Ehrenberg, K.: Der Fundbestand in seiner Gesamtheit. In: Ehrenberg, K. u. a. Die Teufelsoder Fuchslucken bei Eggenburg (NÖ). Denkschr. Ósterr. Akad. Wiss., Math.-Naturwiss. Kl. 112, 137-158, Wien 1966.

Ellermann, J. R. \& Morrison-Scott, T. C. S.: Checklist of Palaearctic and Indian Mammals 1758 to 1946. Brit. Mus. (Nat. Hist.), 810 S., London 1951.

Fuint, W. E.: Die Zwerghamster der paläarktischen Fauna. Die neue Brehm-Bücherei 366, 99 S., $65 \mathrm{Abb}$. , Wittenberg Lutherstadt, (Ziemsen) 1966.

Frenzel, B.: Zur Pollenanalyse von Lössen. Eiszeitalt. u. Gegenw. 15, 5-39, 6 Abb., Ơhringen 1964. - - Die Klimaschwankungen des Eiszeitalters. 291 S., 107 Abb., 23 Tab., Braunschweig 1967.

Guenther, E.: Feinstratigraphische Untersuchungen einer Zwischenschicht und der Deckschichten der Travertine von Ehringsdorf. Alt-Thüringen 3, 1-15, Weimar 1957/58.

HaAse, G.: Stand und Probleme der Lößforschung in Europa. Geogr. Ber. 27, 97-129, Berlin 1963.

Heck, L.: In „Brehms Tierleben“, Bd. 11, 1-576, zahlr. Abb. u. Taf., Leipzig 1914.

Heller, F.: "Die Fauna“... In: Zotz, L.: Das Paläolithikum in den Weinberghöhlen bei Mauern. Quartär-Bibliothek 2, 220-308, Taf. XII-XIV, Bonn 1955.

HePtNER, W. G.: Uber die morphologischen und geographischen Beziehungen zwischen Mustela putorius und Mustela eversmanni. Zeitschr. Säugetierkunde 29, H. 6, 321-330, 4 Abb., Hamburg und Berlin 1964.

Jacobshagen, E., Huckriede, E. \& Jacobshagen, V.: Eine Faunenfolge aus dem jungpleistozänen Löß bei Bad Wildungen. Abh. Hess. Geol. Landesamt f. Bodenforsch. 44, 105 S., 9 Abb., 14 Taf., Wiesbaden 1963.

KAHLKE, H. D.: Die jungpleistozänen Säugetierfaunen aus dem Travertingebiet von TaubachWeimar-Ehringsdorf. Alt-Thüringen 3, 97-130, 10 Abb., Weimar 1957/58.

Koвx, F. E.: Le Putois d'Eversmann fossile en Suisse et en France. Ecl. geol. Helv. 44, no. 2, 394-398, 2 Abb., Basel 1951.

LANG, H.: Erläuterungen z. Geol. Karte v. Niedersachsen $1: 25000$, Bl. Mellendorf. 126 S., 23 Abb., 3 Taf., 1 Kt., Hannover 1967.

Macoun, J. \& RuzickA, M.: The Quaternary of the Upper Moravian Basin in the relation to the Sediments of the Continental glaciation. Anthropozoikum, R. A, 4, 125-166, 16 Abb., 7 Taf., 2 Tab., Prag 1967.

Musı, R.: Über die Möglichkeit der Datierung von Würm-Sedimenten auf Grund von Säugetierfaunen. Rep. VIth Int. Congr. Quatern. Warschau 1961, vol. II, Palaeozool. Sect., 541-545, Lódz 1964.

Musil, R. \& VALOCH, K.: Beitrag zur Gliederung des Würms in Mitteleuropa. Eiszeitalt. u. Gegenw. 17, 131-138, 1 Tab., Öhringen 1966.

Nehring, A.: Über Tundren und Steppen der Jetzt- und Vorzeit mit besonderer Berïcksichtigung ihrer Fauna. Berlin 1890.

OGnew, S. I.: Säugetiere und ihre Welt. 362 S., 110 Abb., 10 Taf., Akademie-Verlag, Berlin 1959. 
Rohdenburg, H. \& Meyer, B.: Zur Feinstratigraphie und Palaeopädologie des Jungpleistozäns nach Untersuchungen an südniedersächsischen und nordhessischen Lößprofilen. In: Lößforschungen in Süiniedersachsen und Nordhessen, Sonderheft z. Tagung der Deutschen Quartärvereinigung. Mitt. D. Bodenk. Ges. 5, 1-135, 25 Abb., Göttingen 1966.

Samson, P. \& Radulescu, C.: Equisse de Stratigraphie würmienne en Roumanie. Rep. VI. Int. Congr. Quarternary, Warschau 1961, vol. 2, Palaeozool. Sect., 577-581, Lódz 1964.

Sibava, V.: Study of the Pleistocene of the glaciated and nonglaciated area of the Bohemian Massif. Anthropozoikum, R. A. 4, 7-36, 19 Abb., 8 Taf., Prag 1967.

Soergel, W.: Lösse, Eiszeiten und die paläolithischen Kulturen. 177 S., 14 Abb., Jena 1919. - Zur biologischen Beurteilung diluvialer Säugetierfaunen. Sitzber. Heidelberger Akad. Wiss., Mathem. Naturwiss. Kl. 4, Abh., 1-37, Heidelberg 1940.

Woldstedt, P.: Das Eiszeitalter. Bd. 1: Allgemeine Erscheinungen des Eiszeitalters. 374 S. m. zahlr. Abb. u. Tab., Bonn 1961. - - Bd. 2: Europa, Vorderasien und Nordafrika im Eiszeitalter. 438 S. m. zahlr. Abb. u. Tab., Bonn 1958.

Wurm, A.: Ưber eine neu entdeckte Steppenfauna von Mauer a. d. Elsenz bei Heidelberg. Jber. u. Mitt. Oberrhein. geol. Ver., N. F. 4, H. 1, 62-78, 2 Taf., Stuttgart 1913.

ZAPFE, H.: Die übrigen Carnivoren (außer Höhlenhyaene und Höhlenbär). In Ehrenberg, K. u. a.: Die Teufelslucken oder Fuchsenlucken bei Eggenburg (N.Ó). Denkschr. Österr. Akad. Wiss., Math. Naturwiss. Kl. 112, 23-38, 3 Abb., Wien 1966.

Žéízko, J. V.: Der Steppeniltis (Foetorius Eversmanni Less.) im Diluvium bei Wolin. Bull. Int. Acad. Sci. Boheme 26, No. 59, II Kl., Prag 1918.

Manuskript eingeg. 10. 7. 1968.

Anschrift des Verf.: Prof. Dr. O. Sickenberg, Niedersächsisches Landesamt für Bodenforschung, 3 Hannover-Buchholz, Alfred-Bentz-Haus. 